\title{
DENSE SENSOR NETWORKS THAT ARE ALSO ENERGY EFFICIENT: WHEN 'MORE' IS 'LESS'
}

\author{
Yao-Win Hong, Anna Scaglione and Rajit Manohar \\ School of Electrical and Computer Engineering, \\ Cornell University, Ithaca, NY 14853 \\ yh84@cornell.edu; anna@ece.cornell.edu; rajit@csl.cornell.edu
}

\section{Abstract ID \#: 858}

\begin{abstract}
In this paper we compare the energy efficiency of wireless sensor networks sampling a continuous sensor field in two different types of deployment, which we refer to as the High Density (HDSN) and the Low Density (LDSN) Sensor Network architectures. In the LDSN, a set of sensors with high resolution are critically deployed at sampling locations so that each sample is nearly uncorrelated and is transmitted to the central node in a separate channel. In HDSN, a simple zero-crossing detector is used at each sensor and the sensor field is reconstructed at the central node with the zero-crossing information extracted from the sensors' $o b$ servations. By proposing a scalable data collection protocol for HDSN, we show that the reconstruction performance of the sensor field at the central processor can be achieved with low complexity at the same bandwidth and energy cost. Therefore, the longevity of the sensors is increased due to the reduced per node energy consumption and the reduced computational energy for the data representation at each sensor. Furthermore, we claim that the system versatility and fault tolerance of HDSN makes it an better alternative to the LDSN architecture.
\end{abstract}

\section{INTRODUCTION}

In recent years, several authors acknowledged the fact that networking is a major obstacle in the development of large scale wireless network. In fact, in the point-to-point network scenario, Gupta and Kumar [1] showed that the per node throughput vanishes as $O(1 / \sqrt{N})$, where $N$ is the number of nodes in the network. Even worse scaling laws were derived for the many-to-one network scenario of $O(1 / N)$ [2] or $O(\log N / N)$ [3] (when applying antenna sharing). These arguments indicate that having a large number of sensors in a wireless network creates such a communication burden that it is crucial to minimize the number of sensors

This work is supported in part by NSF under grant CCR-0431077 and ITR 0428427. needed. In fact, many authors suggest that a dense deployment should be avoided in sensor networks (see e.g. [2]). In this paper we argue the opposite. We show that, if one collects the distributed information to a central node with a data driven strategy, both the communication cost and energy consumption remain the same or decrease as the sensor density increases, provided that the sensor field has limited degrees of freedom in the model. This is especially important in the sensor network application since the sensor field is invariant to the sensor deployment. In our argument we utilize data-driven multiple access protocols that we recently proposed $[4,5]$ requiring a communication cost that scales with the underlying data complexity instead of the number of nodes in the network. Our main objective is to demonstrate that the 'quantity' of the sensors (i.e. a lot of cheap sensors with small batteries) can be effectively traded-off with the 'quality' of each sensor (i.e. few, highly accurate and critically deployed sensors with large energy supplies). Hence, the decision on network deployment does not necessarily have to favor the configuration of highly accurate sensors deployed at the minimum needed density versus a network crowded with cheap devices; instead, it can find the optimum point in terms of energy between these two extremes. The denser network is actually more versatile, because a number of parameters necessary to calculate the optimal deployment of highly capable nodes are not known a-priori.

In our comparison we examine two extreme examples of network architecture: (i) The High Density Sensor Network (HDSN), where cheap sensors are deployed with high density; (ii) The Low Density Sensor Network (LDSN), where a small number of high resolution sensors are critically deployed at the minimum sampling points in the sensor field. The comparison is done at three fundamental levels: 1) the reconstruction performance attainable by the two systems considering a simple zero-crossing detector in the HDSN and a high precision A/D converter in the LDSN; 2) the comparison between the energy requirements for the data collection; 3) the energy required by the hardware nec- 
essary to implement the two architectures.

The energy efficiency of the data collection process is discussed in two parts. First, we describe the data-driven transmission scheduling scheme that extracts the data efficiently from a large population of sensors and show that the same reconstruction performance can be obtained by utilizing the same bandwidth resources for both the HDSN and the LDSN. Secondly, we compare in terms of transmission power where we show that the two systems have comparable power consumption when they utilize transmission methods with comparable complexity.

\section{PROBLEM SETUP}

Consider a network of sensors where each sensor takes measurements from a real continuous scalar field defined over the $m$-dimensional spatial coordinates represented by the vector $\mathbf{x}$, and the time-dimension $t$. Assume that the field is expanded (approximately) in a finite dimensional basis, i.e. the field $V(\mathbf{x}, t)$ is such that:

$$
V(\mathbf{x}, t)=\sum_{k=1}^{M} C_{k} \psi_{k}(\mathbf{x}, t) .
$$

where $\psi_{k}(\mathbf{x}, t), k=1, \cdots, M$ is a set of $m$-dimensional independent basis functions. $M$ is effectively the number of degrees of freedom of the sensor field. The expansion in (1) can be valid assuming a deterministic model or can be interpreted in the mean square sense for a random model. Our goal is to reconstruct the field remotely with a certain Mean Square Error (MSE) from a finite set of quantized measurements. In Fig. 1, we illustrate a one-dimensional network of sensors observing a smooth random signal in both space and time as defined in (1). The grey flat plane in the figure is the zero-plane, which highlights the zero-crossing contours of the sensor field (black mesh curve). In LDSN (the cross-headed arrows), the sensors are deployed at the critical sampling points where an accurate data representation of each sample is transmitted to the central processor. In contrast, in the HDSN the sensors are densely deployed while each sensor records only the local zero-crossing points in time (1 bit quantization).

\section{Sampling with infinite precision}

Let $\mathcal{D}=\left\{\left(\mathbf{x}_{i}, t_{i}\right), i=1, \ldots, K\right\}$ be the reconstruction set that represents the set of sampled values utilized for reconstruction. Clearly, if the sensors do not move, several samples in time are taken at the same locations in space.

\section{(1) Explicit sampling}

Let $\mathbf{V}$ be the vector of samples with infinite precision at the points in $\mathcal{D}$, i.e. $(\mathbf{V})_{i}=V\left(\mathbf{x}_{i}, t_{i}\right)$ where $\left(\mathbf{x}_{i}, t_{i}\right) \in \mathcal{D}$.

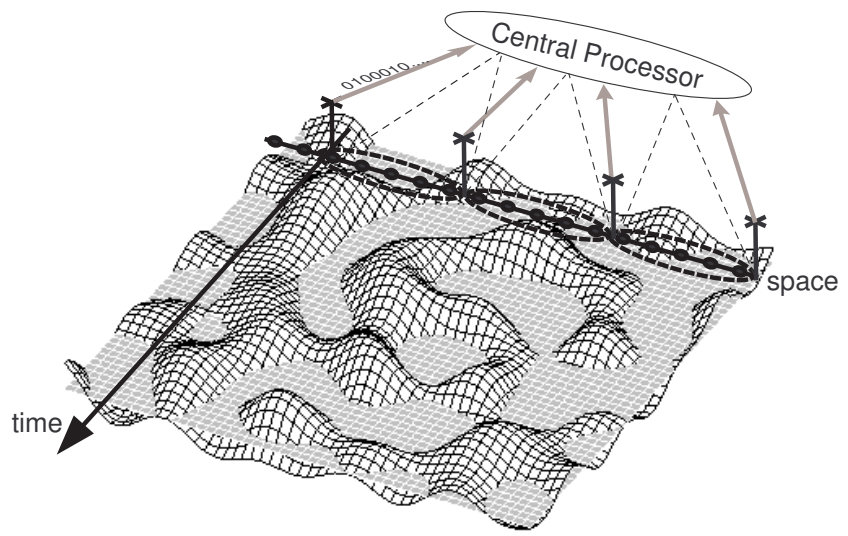

Fig. 1. Illustration of the HDSN (black points) versus the LDSN (cross-headed points) sampling in time.

Definition 1 Suppose there exists $\mathcal{D}$ such that the matrix

$$
\mathbf{A}:(\mathbf{A})_{i, k}=\psi_{k}\left(\mathbf{x}_{i}, t_{i}\right),
$$

is invertible. Such a set $\mathcal{D}$ is defined to be a minimum reconstruction set if and only if, for all other sets $\mathcal{D}^{\prime}$ that satisfy the invertibility criterion of (2), we have $\left|\mathcal{D}^{\prime}\right| \geq|\mathcal{D}|$.

Note that a minimum reconstruction set is not necessarily unique. Furthermore, among the minimum reconstruct sets the one to be preferred is such that $\mathbf{A}$ has minimal condition number, which minimizes the estimation error in the presence of noisy measurements. Clearly, we have $|\mathcal{D}| \geq M$. Denoting by $\mathbf{C}$ the vector of the coefficients in (1), it is clear that $\mathcal{D}$ guarantees the existence of the solution $\mathbf{C}=\mathbf{A}^{\dagger} \mathbf{V}$ († stands for pseudo inverse) and a perfect reconstruction of $V(\mathbf{x}, t)$ is attainable at all points using (1). (2) Implicit sampling

The implicit sampling here refers to the dither-sampling method proposed in [6]. In this case, the field $V(\mathbf{x}, t)$ is represented with infinite precision at the set of coordinates

$$
\mathcal{V}_{g}=\left\{\left(\mathbf{x}_{i}, t_{i}\right): V\left(\mathbf{x}_{i}, t_{i}\right)-g\left(\mathbf{x}_{i}, t_{i}\right)=0\right\} .
$$

where $g$ represents the dither function. Similar to the previous case, exact reconstruction is possible if $\mathcal{V}_{g}$ contains a minimum subset $\mathcal{D} \subseteq \mathcal{V}_{g}$ as defined in Definition 1. Denote by $\mathbf{g}$ the vector $[\mathbf{g}]_{i i}=g\left(\mathbf{x}, t_{i}\right)$, it follows that

$$
\mathbf{A C}=\mathbf{V}=\mathbf{g} \quad \rightarrow \quad \mathbf{C}=\mathbf{A}^{\dagger} \mathbf{g}
$$

\section{Sampling with finite precision}

In practice, the sampling cannot be achieved with infinite precision either due to the finite levels of quantization or because of the finite deployment of sensors. The quantization error is on the sampled value $\hat{\mathbf{V}}=\mathbf{V}+\mathbf{E}_{V}$ for $e x$ plicit sampling and on the level crossing time and position $\hat{\boldsymbol{p}}_{i}=\left(\hat{\mathbf{x}}_{i}, \hat{t}_{i}\right)=\boldsymbol{p}_{i}+\boldsymbol{\tau}_{i}$ for implicit sampling. 
Let us assume that, with finite precision (explicit or implicit) sampling, we have an estimate $\hat{\mathbf{C}}=\mathbf{C}+\mathbf{E}_{C}$. Thus, the sensor field reconstruction and its MSE are respectively:

$$
\begin{gathered}
\hat{V}(\mathbf{x}, t)=V(\mathbf{x}, t)+\sum_{m=1}^{M}\left[\mathbf{E}_{C}\right]_{m} \psi_{m}(\mathbf{x}, t) \\
D=\int E\left\{\left|\sum_{m=1}^{M}\left[\mathbf{E}_{C}\right]_{m} \psi_{m}(\mathbf{x}, t)\right|^{2}\right\}(d \mathbf{x}) d t=\operatorname{tr}\left(\mathbf{\Psi} \mathbf{R}_{E_{C}}\right)
\end{gathered}
$$

where $\mathbf{R}_{E_{C}}=E\left\{\mathbf{E}_{C} \mathbf{E}_{C}^{T}\right\}$ and $\operatorname{tr}(\mathbf{X})$ is the trace of matrix $\mathbf{X}$ and $\boldsymbol{\Psi}$ has $(m, n)$-th element defined as

$$
[\mathbf{\Psi}]_{m, n}=\int \psi_{m}(\mathbf{x}, t) \psi_{n}(\mathbf{x}, t)(d \mathbf{x}) d t .
$$

Although the imprecision in the estimated coefficient $\mathbf{E}_{C}$ exists in any case, the quantization error in explicit and implicit sampling contributes differently to $\mathbf{E}_{C}$ and, consequently, to $D$. In fact, in the case of explicit sampling $\hat{\mathbf{V}}=\mathbf{V}+\mathbf{E}_{V}$ and, thus:

$$
\mathbf{E}_{C}=\mathbf{A}^{\dagger} \mathbf{E}_{V} \Rightarrow D=\operatorname{tr}\left(\boldsymbol{\Psi} \mathbf{A}^{\dagger} \mathbf{R}_{E_{V}}\left(\mathbf{A}^{\dagger}\right)^{T}\right)
$$

Because the entries of $\mathbf{E}_{V}$ are in the order of the quantization step-size $q$, it follows from (7) that $D=O\left(q^{2}\right)$.

Omitting the details for brevity, in the case of implicit sampling, a first order approximation of the error is:

$$
\begin{gathered}
\mathbf{E}_{C}=\mathbf{A}^{\dagger}\left(\mathbf{E}_{g}-\mathbf{E}_{A} \mathbf{C}\right) \\
{\left[\mathbf{E}_{g}\right]_{i}=\nabla g\left(\mathbf{x}_{i}, t_{i}\right) \boldsymbol{\tau}_{i}, \quad\left[\mathbf{E}_{A}\right]_{i, m}=\nabla \psi_{m}\left(\mathbf{x}_{i}, t_{i}\right) \boldsymbol{\tau}_{i} .}
\end{gathered}
$$

Assuming that the maximum quantization-step in the coordinates $\left(\hat{\mathbf{x}}_{i}, \hat{t}_{i}\right)$ is $\tau$, also in this case because $\mathbf{E}_{g}$ and $\mathbf{E}_{A}$ entries are proportional to $\tau$, evidently $D=O\left(\tau^{2}\right)$.

To streamline our discussion in the following we restrict ourselves to the one-dimensional case where the underlying sensor field is bandlimited. The same arguments can be rephrased to handle a multi-dimensional, space and time, sampling problem.

\section{EQUIVALENCE OF THE RECONSTRUCTION PERFORMANCE}

When the underlying sensor field can be described by a onedimensional bounded function $V(x) \in \mathcal{B}_{2}^{W}$, where $\mathcal{B}_{2}^{W}$ is the set of all bandlimited functions in $L^{2}(\mathbf{R})$ that are bounded in frequency by $W \leq 1$ without loss of generality (i.e. the Fourier Transform $\mathcal{F} V(\omega)=0$ for $|\omega|>W$ and $\left.\|V\|_{2}<\infty\right)$ the basis function in (1) is the sinc basis $\psi_{m}(x)=\sin (\pi(x-m)) /(x-m)$. The $N$ sensors

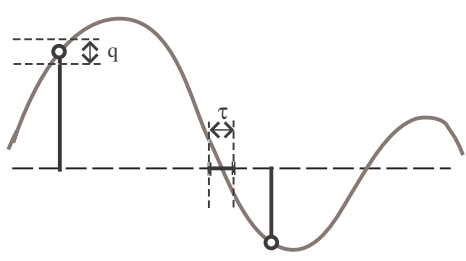

Fig. 2. The illustration of the quantization resolution $q$ and the zero-crossing resolution $\tau$.

$\mathcal{S}=\left\{s_{0}, \cdots, s_{N-1}\right\}$ are in this simplified model deployed in a one-dimensional network and let $x_{i} \in \mathcal{D}$ be the location of sensor $s_{i}$ such that $x_{0}<x_{1}<\cdots<x_{N-1}$.

\section{(1) Low Density Sensor Networks \& Explicit sampling}

In LDSN, the sensors are critically deployed with equal spacing $d=1 / 2 \lambda W$ (i.e. the sampling period for a $W$ bandlimited function with the over-sampling ratio $\lambda>1$ ), where each sensor observes the Nyquist sample, quantized with a uniform quantizer having quantization error less than $q$ as shown in Fig. 2. The mean square (MS) total distortion in this case is approximately [7]

$$
D=\frac{M}{12 \lambda} q^{2} .
$$

As shown in [8], comparable reconstruction performance can be using the knowledge of the zero-crossing points.

\section{(2) High Density Sensor Networks \& Implicit sampling}

In HDSN, we assume that the distance between adjacent sensors is less than $\delta$, i.e. $\left|x_{i}-x_{i+1}\right|<\delta$, for $\delta$ small. By recording the zero-crossing instants at each sensor position, the sensors have knowledge of the sign information at the particular snapshot in time, i.e.

$$
\hat{V}_{i}=\operatorname{sgn}\left[V\left(x_{i}\right)\right]
$$

where $\operatorname{sgn}(y)=1$ if $y \geq 1$ and $\operatorname{sgn}(y)=0$ otherwise. If there exists two sensors $s_{i}$ and $s_{j}$ such that $\hat{V}_{i} \neq \hat{V}_{j}$ and $\left|x_{i}-x_{j}\right|<\tau$, then the precision of the zero-crossing point can be obtained up to $\tau$, as shown in Fig. 2. In this case, it has been shown in [8] that the distortion of the zero-crossing reconstruction is bounded deterministically as

$$
|V(x)-\hat{V}(x)|^{2}<c_{v} \tau^{2}
$$

when the crossings are obtained for a sequence of stable sampling points [8], which provide a valid reconstruction set $\mathcal{D}$. Hence, LDSN and HDSN achieve a comparable MS distortion performance if $q$ is of the order of $\tau$.

Note that it is necessary to choose the dithered function $g(x)$ such that there is at least one zero-crossing point within each Nyquist sampling period. For example, for 


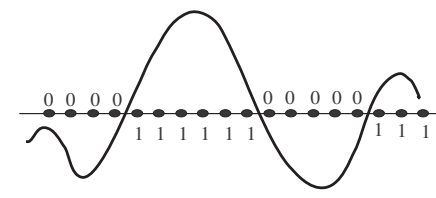

Fig. 3. Long patches of zero and one in recording the sign information of the bandlimited field.

$V \in \mathcal{B}_{2}^{W}$ and $|V|<1$, the function $g(x)=\gamma \cos (2 \pi W \lambda x)$, where $\gamma>1$, is sufficient to achieve the sequence of stable sampling points $[6,9]$. The reconstruction is achieved through the inversion of $\mathbf{A}$, where the invertibility is guaranteed.

\section{EQUIVALENCE OF THE COMMUNICATION RESOURCES}

We calculate the data collection cost for LDSN and HDSN respectively as a function of the number of channel accesses required and the total power needed to deliver the necessary information, for equivalent reconstruction performance. To obtain comparable communication cost in HSDN we gather the zero crossing information using a generalized data driven strategy for data collection known as Group Testing Multiple Access (GTMA).

\section{Group Testing Multiple Access}

If $V(x)$ is a smooth function, it is likely that large patches of sensors in the proximity of each other will observe the same sign information $\left(\operatorname{sgn}\left(V_{i}\right)=0\right.$ or $\left.\operatorname{sgn}\left(V_{i}\right)=1\right)$ as shown in Fig. 3. Because the data are highly redundant and because we are only interested in finding one zero crossing per Nyquist interval, it would be extremely inefficient to poll each sensor individually. In GTMA, the channel assigned to a specific group of sensors $U \subset \mathcal{S}$ : each sensor in the group answers simultaneously to a query based on its own data (in this case based on $\operatorname{sgn}\left(V_{i}\right)$ ) [4]. Depending on the query, this may require one or more channel uses. The subclass of GTMA strategies that is effective in our problem is the so called tree splitting strategy, where the groups of sensors scheduled to transmit are in adjacent locations ${ }^{1}$ and the query comprises two questions (and thus two channel uses): 'do you have $\operatorname{sgn}\left(V_{i}\right)=0$ ?' and 'do you have $\operatorname{sgn}\left(V_{i}\right)=1$ ?'. When both queries receive a positive answer, we have an erasure $e$ that indicates the presence of a zero crossing in that group. Fig. 4 illustrates the treesplitting strategy. GTMA queries the entire spatial Nyquist

\footnotetext{
${ }^{1}$ We assume that each sensor has hard-coded its own position when deployed and that knows in which location it is.
}

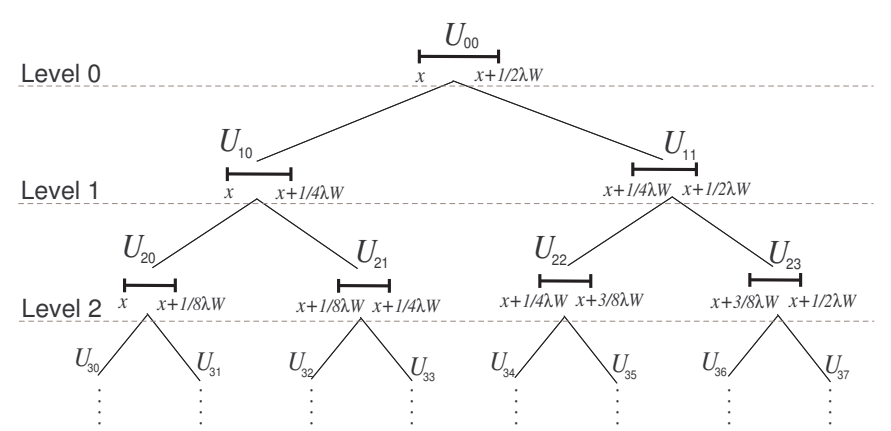

Fig. 4. The illustration of the tree algorithm by spatially dividing the one-dimensional regions.

interval $[x, x+1 / 2 \lambda W)$, where $x$ is a Nyquist sampling point, which we call group $U_{00}$. If an " $e$ " is observed, the interval is adaptively divided into subintervals until a " 0 ", or " 1 " is observed. At each level of the tree, the interval is separated into disjoint intervals having equal length. For instance, at level $k$ the interval is divided into $2^{k}$ disjoint intervals. This process locates zero-crossings using the minimum number of channel accesses [4] which in this case is $k=O\left(\log \frac{1}{\tau}\right)$, where $\tau$ is the desired precision. Since it is guaranteed by the dither function that at least one zerocrossing point occurs within each Nyquist interval, we can consider the first answer to be an erasure and start splitting immediately.

In the multidimensional case, we envision the HDSN to be activity driven, i.e. when the information relative to the current Nyquist interval in time and space has been collected, the network falls asleep until the Nyquist interval expires. After that, the sensors wake up and as soon as a node records a crossing, it sends a broadcast signal which is replicated cooperatively at the physical layer. The problem of collisions is ruled out, because other nodes that transmit their zero crossing information nearly simultaneously, only reinforce the signal that initiates the GTMA.

\section{Communication Cost of HDSN versus LDSN}

In LDSN, an obvious choice for the transmission scheduling is to enable the critically deployed sensors to transmit their encoded samples through independent channels since the data at each sensor are non-redundant. In this case, the number of channel accesses for each sensor is equal to $k=O\left(\log \frac{1}{q}\right)$ when there are $k$ levels in the quantizer, i.e. the quantization step $q=c \cdot 2^{-k}$ where $c$ is a constant depending on the dynamical scale of the signal or the desired distortion at the receiver.

In the tree splitting protocol used for HDSN, only one group of sensors is scheduled to transmit in each level of the 
tree shown in Fig. 4. Therefore, the zero-crossing point is located within an interval of length $\tau=2^{-k} / 2 \lambda W$ by using only $k$ number of channel accesses where $k=O\left(\log \frac{1}{\tau}\right)$. Hence, the information required for reconstruction in both LDSN and HDSN can be retrieved with the same number of channel accesses.

\section{ENERGY EFFICIENCY OF HIGH DENSITY SENSOR NETWORKS}

We consider the simple transmitter cooperation technique proposed in $[10,11]$, for which the power consumption in transmission is show to be equal in HDSN as in LDSN and discuss a second option which is to uses beamforming, where HDSN has a considerable gain over LDSN.

\section{Asynchronous Cooperation for HDSN}

In GTMA, the knowledge of the $0,1, e$ information is sufficient for each sensor to determine whether or not it belongs to the scheduled group of sensors. We assume that the nodes are simple pulse emitters and that in order to distribute output information throughout the network, they use the symbol-by-symbol relay scheme proposed in [10], called the Opportunistic Large Array (OLA) system.

Specifically, let $U$ is the group of sensors that are scheduled to transmit and $U_{b} \equiv\left\{s_{i} \in U: \operatorname{sgn}\left(V_{i}\right)=b\right\} \subset U$ where $\operatorname{sgn}\left(V_{i}\right)=0,1$ is the symbol observed by sensor $s_{i}$ and $b=0,1$. During each transmission period, we assign two independent time slots $(\mathrm{CH} 0$ and $\mathrm{CH} 1)$ to the sensors in $U$. All the sensors in $U_{0}$ emit a pulse $p(t)$ in the channel $\mathrm{CH} 0$ and all the sensors in $U_{1}$ emit a pulse in $\mathrm{CH} 1$. Let us consider the receiver model for $\mathrm{CHO}$ in the following since the signal in $\mathrm{CH} 1$ performs exactly in the same way. Initially, let each sensor in $U_{0}$ emit a pulse $p(t)$, e.g. the mono-cycle pulse in UWB systems, to all other sensors in the network. The signal received at sensor $s_{i}$ is

$$
r_{i}^{(1)}(t)=u_{i}^{(1)}(t)+n_{i}(t)=\sum_{j: s_{j} \in U_{0}} \sqrt{\frac{P_{j}}{d_{j i}^{2}}} p\left(t-d_{j i} / c\right)+n_{i}(t)
$$

where $P_{j}$ is the power emitted by sensor $s_{j}, d_{j i}$ is the distance between $s_{j}$ and $s_{i}, c$ is the speed of light and $n_{i}(t)$ is the noise at sensor $s_{i}$ with variance $N_{o} / 2$.

Although the signal of the sensors in $U_{0}$ will be received by all other nodes, not all sensors will yield a reliable detection. Using the Neyman-Pearson criterion, we define the decision rule at sensor $s_{i}$ as

$$
\mathcal{D}\left(r_{i}^{(1)}(t)\right)=\mathbf{1}_{\left\{\left\|r_{i}^{(1)}(t)\right\|^{2} \geq \nu\right\}}
$$

where $\nu$ is the optimal decision threshold determined through the given false alarm probability. If a pulse is detected in both time slots, the output corresponds to the erasure case while if the pulses are detected in only one time slot, the output corresponds either 0 or 1 . When the area of the network is small, the emitted pulses can be received directly from the transmitting source. If this is not the case, one must rely on neighboring nodes to serve as relays to distant nodes. Let $L_{1} \equiv\left\{s_{i} \in \mathcal{S}-U_{0}: \mathcal{D}\left(r_{i}^{(1)}(t)\right)=1\right\}$ be the set of nodes that received reliably directly from the transmission of the source. All the nodes in this set will then retransmit the same pulse $p(t)$ to all the other sensors in the network, which will then ignite another cooperative relay transmission by the third tier of receivers. This on-going process will continue until all the sensors in the network are reliably informed that there exists a sensor that observed the bit 0 , i.e. $U_{0}$ is non-empty.

Let $L_{l}$ be the set of nodes that received sufficient signal energy to exceed $\nu$ only after the emission of nodes in $U_{0}$, $L_{1}, \cdots, L_{l-1}$. In this case, The signal received by nodes in the $l$-th layer is approximately

$$
r_{i}^{(l)}(t) \approx \sum_{k: s_{k} \in L_{l-1}} \sqrt{\frac{P_{k}}{d_{k i}^{2}}} p\left(t-d_{k i} / c+\sum_{h=1}^{l-1} \delta_{h}\right)+n_{i}(t) .
$$

Not that this methods floods the HDSN with all the data that are necessary to follow the protocol as well as informing the remote central processor. Therefore, coordinating the sensors and reaching the distant receiver are obtained in one operation, at no additional cost.

The results in [11] provide the necessary conditions on the transmitted energy to guarantee full collaboration in the two-dimensional network that guarantee that the network reliably detect and relay the transmitted symbol. We can extend those arguments in the 1-D setup. We denote by $P_{s}$ the power of the source that initiates the transmission and let $P_{r}$ be the power retransmission and assume that the pulse is sufficiently narrow that their contributions can be resolved and combined. We assume that all sensors have the detection threshold $\nu_{c}$ and let the path loss be defined as $1 /(1+d)^{2}{ }^{2}$ The result is provided for a continuum approximation [11] of a dense OLA network with only one source initiating the transmission.

\section{Lemma 1 For a fixed detection threshold $\nu_{c}$, the relay trans-}

\footnotetext{
${ }^{2}$ The additive constant 1 in the denominator of the path loss model guarantees that the channel gain does not go to infinity when the distance is close to zero. However, the performance guaranteed in this scenario will also be achievable for the case where the path loss is $1 / d^{2}$, since the loss in our model decays faster with distance. Furthermore, when $d$ is large, we have $1 / d^{2} \approx 1 /(1+d)^{2}$.
} 
mission is received reliably at an arbitrarily large network area when the sensors are distributed in continuum and if $P_{s}>\nu_{c}$ and $\rho>(3+2 \sqrt{2}) \nu_{c}$, where $\rho$ is the average power emitted by the sensors located within a unit interval.

Let $d_{c}$ be the average distance between the sensor and the central processor, which is common for all nodes if $d_{c} \gg$ $d_{i j}$ for all $i, j$. Assume that the sensor network is confined within the region $[0, D]$, then the minimum power required by each relaying node is $P_{r}=(3+2 \sqrt{2}) \nu_{c} D /(N-1)$. On the other hand, when the full network cooperates, since it is necessary to have $P_{t o t} /\left(1+d_{c}\right)^{2} \geq \nu_{c}$ the required aggregate energy is $P_{t o t}=\nu_{c}\left(1+d_{c}\right)^{2}$. Thus, the power emitted by each relaying sensor should be equal to $P_{r}=$ $\left[\nu_{c}\left(1+d_{c}\right)^{2}-P_{s}\right] /(N-1)$, where $N$ is the number of sensors in the network. In this case, if the condition

$$
\nu_{c}\left(1+d_{c}\right)^{2}-P_{s}>(3+2 \sqrt{2}) \nu_{c} D
$$

holds, the power emitted by the sensors to reach the far destination will simultaneously fulfill the power requirements to achieve sensor collaboration. By setting $P_{s}=\nu_{c}$ (i.e. the minimum source power) and for sufficiently large $D$, the condition is satisfied with $d_{c}>[(3+2 \sqrt{2}) D]^{-1 / 2}$ which is a reasonable distance for a central processor at a far destination. In fact, it is sufficient to have $d_{c}>D$ if $D>3+2 \sqrt{2}$ meters, which is a fairly small area for the deployment of sensor networks and the problems that arise in large scale networks would not be a concern in this scenario.

Remark - The OLA system utilized for comparison in our paper is an asynchronous cooperation strategy which is simple requires minimum overhead for coordination and reduced latency due to the symbol-based relaying structure. However, the receiver performance can be further improved if a more sophisticated cooperation strategy is used. In the extreme case, the sensors can be treated as a large antenna array that performs beam-forming towards the far destination. In this case, for a high density network where $M$ sensors are located in each Nyquist interval, the beam-forming signal achieves $M$ times the gain in the received signal-tonoise ratio as opposed to having one sensor per Nyquist interval in LDSN, which shows that the high density deployment can be superior to LDSN by order of magnitudes, if the complexity of beam-forming towards the central node was tolerable.

\section{Energy Consumption of LDSN}

In LDSN, each sensor transmits their messages to the central processor without cooperation which requires a power of $\nu_{c}\left(1+d_{c}\right)^{2}$ at each sensor. Therefore, the battery at each sensor will be depleted rapidly which would require batteries with high energy density and, thus, high cost.
We should note that, in HDSN, the signals may not be completely orthogonal as assumed previously and the fading may cause destructive interference. Nevertheless, it is expected that in the high density network the per node energy consumption is still reduced significantly.

\section{THE COST OF HARDWARE AND COMPUTATION}

The local cost of sampling and computation for the two mechanisms has many contributions. First, the physical phenomenon of interest must be sensed. Assuming that the result of the sensing process is a signal that must be digitized, we estimate the cost of creating the sample as the cost of analog-to-digital (A/D) conversion. Once the sample is created, there is an additional energy cost in converting the sample into a form suitable for communication, and the associated protocol processing overhead associated with different communication mechanisms. We will estimate the algorithmic overhead of the two mechanisms assuming a highly optimized platform for the sensor nodes $[13,14]$.

\section{(1) Analog-to-digital conversion}

A useful figure-of-merit for A/D converters is given by $F=2^{S N R b i t s} \times f_{\text {samp }} / P_{\text {dis }}$, where $S N R$ Rits is the name conventionally used to indicate the number of bits of the A/D converter, $f_{\text {samp }}$ is the sampling frequency, and $P_{\text {dis }}$ is the dissipated power. This metric combines the sampling frequency, the precision, and the power dissipation of the A/D converter allowing comparison across different implementations [12]. In other words, given a fixed sampling rate and architecture, the power requirements for an A/D should scale with the number of quantization levels. For critically deployed LDSNs, the power required to generate a sample will be dependent on $1 / q$, where $q$ is the quantization uncertainty. For the HDSN scenario with simple zero-crossing detectors, the power requirements will be independent of $q$-in fact, they should be a factor $1 / q$ lower than the A/D converter required for the HDSN. The frequency of sampling, $f_{\text {samp }}$ for the LDSN deployment is given by the Nyquist rate, and is fixed by the bandwidth of the power spectral density of the random process in time. For HDSN, the samples are generated in an irregular fashion by the random zero crossings that occur. Hence, the parameter $f_{\text {samp }}$ is replaced by the expected number of crossings of the random process. For example, using the celebrated Rice's formula, for a stationary Gaussian process the average number of crossings is equal to $1 / \pi \sqrt{-\rho^{\prime \prime}(0)}$, where $\rho(\tau)$ is the correlation coefficient of the process. Interestingly, $-\frac{1}{\pi^{2}} \rho^{\prime \prime}(0)=\frac{\int_{-\infty}^{\infty} 4 f^{2} S(f) d f}{\int_{-\infty}^{\infty} S(f) d f}$. This is of the order of the normalized second moment of the power spectral density. The square root of this is analogous to a stan- 
dard deviation, and is a measure of the support of $S(f)$ in the frequency domain. Hence, on average, the number of crossings should be of the same order as expected given the Nyquist rate.

The net effect is that while both schemes generate the same number of samples, the HDSN scenario uses much less power to generate each sample-a factor of $1 / q$ lower.

\section{(2) Protocol overhead}

The protocol overhead is a quantity that is difficult to provide asymptotic arguments for, as it depends on the precise nature of the underlying hardware architecture. The bulk of the power contribution is from the actual protocol execution. For both LDSN and HDSN the number of protocol invocations is of the same order, because both methods only report information when there is a non-redundant sample. The additional activity in the HDSN case comes from the fact that more nodes are involved in relaying messages.

While for the LDSN a clocked design is the most suitable, for the HDSN we can assume an optimized activitydriven computation platform where the sensor node can: 1) efficiently sleep when it is not performing any useful work; 2) have extremely efficient transitions to/from active and idle states; 3) have low software overhead from the operating system [14]. The SNAP/LE platform we have developed encompasses all these features and require nanowatts of additional power to perform the computation [14]. In a standard CMOS process, the amount of power required when a node is sleeping is larger than a hundred nanowatts, and therefore the minor additional energy required for relaying is less than the power dissipation of a sleeping node!

The net effect is that both mechanisms should require the same amount of power for handling the communication protocols.

\section{THE SYSTEM VERSATILITY OF HIGH DENSITY SENSOR NETWORKS}

In the LDSN, the critical deployment of sensors at Nyquist sampling points will limit their applications for sensor fields that are bandlimited to frequencies lower than that of the corresponding Nyquist sampling rate. Also, the failure of any sensor would cause a large distortion in the signal reconstruction. Hence, the LDSN is not robust and lacks adaptivity to different applications and to hostile environments. One may argue that redundant sensors can be deployed densely in the network to introduce robustness and that the communication congestion can be avoided by choosing only the sensors at the Nyquist sampling points to transmit their information. However, the high resolution sensors utilized in the LDSN are extremely costly and it would be wasteful to distribute these sensors in a large scale.
In contrast to the LDSN, the advantage of HDSN is its system versatility and robustness to failure. In fact, the failure of a node will not increase the distortion as drastically as that of LDSN. Furthermore, if the density of the nodes are sufficiently high. One can simply increase the number of channel access to increase the precision of the zero-crossing position as illustrated in the tree splitting algorithm. Similarly, one can increase the number of channel accesses to retrieve additional zero-crossing points, adjusting to the frequency of the sensor field. Therefore, the network configuration is invariant to the application at hand.

\section{REFERENCES}

[1] P. Gupta and P. R. Kumar, "The capacity of wireless networks," IEEE Trans. Inform. Theory, vol. IT-46, no. 2, pp. 388-404, Mar. 2000.

[2] D. Marco and D. L. Neuhoff, "Reliability vs. efficiency in distributed source coding for field-gathering sensor networks," in Proc. on the Third International Symposium on Information Processing in Sensor Networks (IPSN), Apr. 2004.

[3] H. E. Gamal, "On the scaling laws of dense wireless sensor networks," to appear in IEEE Trans. on Inform. Theory, 2005.

[4] Y.-W. Hong and A. Scaglione, "On multiple access for correlated sources: A content-based group testing approach," in Proc. of IEEE ITW, San Antonio, TX, Oct. 2004.

[5] — , "Content-based multiple access: Combining source and multiple access coding for sensor networks," in Proc. of IEEE MMSP, Siena, Italy, Sept. 2004.

[6] Z. Cvetković and I. Daubechies, "Single-bit oversampled a/d conversion with exponential accuracy in the bit-rate," in Proc. DCC, Mar. 2000, pp. 343 - 352.

[7] W. R. Bennett, "Spectra of quantized signals," Bell System Technical Journal, vol. 27, pp. 446-472, July 1948.

[8] Z. Cvetković and M. Vetterli, "On simple oversampled A/D conversion in L2(R)," IEEE Trans. Inform. Theory, vol. 47, no. 1, pp. 146-154, Jan. 2001.

[9] Z. Cvetković, I. Daubechies, and B. F. Logan, "Interpolation of bandlimited functions from quantized irregular samples," in Proc. DCC, Apr. 2002, pp. 412-421.

[10] A. Scaglione and Y.-W. Hong, "Opportunistic large arrays: Cooperative transmission in wireless multi-hop ad hoc networks for the reach back channel," IEEE Trans. Signal Proc., vol. 51, Aug. 2003.

[11] B. Sirkeci-Mergen and A. Scaglione, "A continuum approach to dense wireless networks with cooperation," in Proc. of INFOCOM 2005, Miami, FL, Mar. 2005.

[12] Robert H. Walden. "Analog-to-Digital Converter Survey and Analysis," IEEE Journal on Selected Area in Communications, vol. 17, Apr. 1999.

[13] C. Kelly, V. Ekanayake, and R. Manohar. "SNAP: A Sensor Network Asynchronous Processor," Proc. ASYNC 2003, Vancouver, BC, May 2003.

[14] V. Ekanayake, C. Kelly, and R. Manohar. "An Ultra Low Power Processor for Sensor Networks," Proc. of ASPLOS 2004, Boston, MA, October 2004. 